\title{
A Vertical Monolithic Combination of an InGaAsP/ InP Laser and a Heterojunction Bipolar Transistor
}

\author{
T. R. CHEN, KATSUYUKI UTAKA, MEMBER, IEEe, YUHUA ZHUANG, YA-YUN LIU, \\ AND AMNON YARIV, FELLOW, IEEE
}

\begin{abstract}
A DH InGaAsP / InP mesa laser and a DH InGaAsP / InP mass-transport laser were successfully put together with an InGaAsP / InP heterojunction bipolar transistor in a vertical configuration. $\mathrm{A}$ laser threshold current as low as $17 \mathrm{~mA}$ and an output laser power of over $30 \mathrm{~mW}$ were achieved. Base injection current-controlled optical bistability and optical switching were demonstrated.
\end{abstract}

\section{INTRODUCTION}

$\mathrm{I}^{\mathrm{N}}$ $\mathrm{N}$ recent years, there has been continuous interest in developing integrated optoelectronic circuits (IOEC). This is mainly for its potential towards achieving compact, high-speed, high-reliability, multifunctional devices.

The InGaAsP / InP system is of great interest in longhaul, high bit rate optical communications. There are increasing efforts in monolithically integrating a laser diode (LD) or light-emitting diode (LED) with a heterojunction bipolar transistor (HBT) [1]-[5]. The reason for using an HBT instead of the more usual FET is that bipolar devices are potentially faster, less noisy at high frequencies, while yielding better device-to-device uniformity [6]. Moreover, the HBT benefits from heteroepitaxy technology in the same way as the double-heterostructure laser, i.e., by using the energy gap variations in addition to electric fields, within wide limits, to control the distribution and flow of electrons and holes independently [7]. In a double-heterostructure bipolar transistor, the interchangeability of collector and emitter provides a further degree of freedom in IC design. Still another advantage of HBT's is that they are more versatile than FET's. For example, an HBT can be operated as a phototransistor [1]. A laser or LED integrated with HBT's can, therefore, be controlled either electronically or optically. In practice, as will be shown in this paper, the technology for making LD's and HBT's is often compatible, while high-performance InP FET's are not yet available.

\footnotetext{
Manuscript received October 15, 1986; revised February 18, 1987. This work was supported by the U.S. Office of Naval Research (L. Cooper), the National Science Foundation (K. Gustafson), and the U.S. Army Research Office (R. Guenther).

T. R. Chen and Y. Zhuang are with the Chengdu Institute of Radio Engineering, China, on leave at the Department of Applied Physics, California Institute of Technology, Pasadena, CA 91125.

K. Utaka was on leave at the Department of Applied Physics, California Institute of Technology, Pasadena, CA 91125 . He is with the KDD Research and Development Laboratories, Meguro-ku, Tokyo 153, Japan.

Y.-Y. Liu and A. Yariv are with the Department of Applied Physics, California Institute of Technology, Pasadena, CA 91125.

IEEE Log Number 8714531.
}

There have been several works on the integration of an InGaAsP / InP LD (or LED) with HBT's [1]-[5]. In a planar geometry [5], a buried heterostructure InGaAsP/InP laser and three HBT's were integrated on a single InP substrate. However, in a vertical configuration [1]-[4], only LED's have been successfully integrated with HBT's.

In this paper, we report on a monolithic vertical device consisting of a DH InGaAsP / InP laser and a heterojunction bipolar transistor on $\mathrm{n}^{+}-\mathrm{InP}$ substrates. With this true three-terminal device, lasing action, driven and controlled electronically by the HBT, was achieved. Electrooptical switching and bistability were also demonstrated.

Section II gives a brief description of the device structure and operation principles. In Section III, the fabrication and device characteristics of the HBT are presented. Section IV describes the fabrication process of the monolithic device, and Section V summarizes the performance of the monolithic LD/HBT device.

\section{Structure and Operation Principles}

A schematic structure of the monolithic device is shown in Fig. 1. The structure consists of seven epitaxially grown layers. The top four layers, i.e., the n-InP lower confining layer, undoped InGaAsP active layer, p-InP upper confining layer, and $\mathrm{p}^{+}-\mathrm{InGaAsP}$ contact layer, form a conventional DH laser. The bottom four layers, i.e., the $\mathrm{n}^{+}$-InP emitter layer, $\mathrm{p}$-InGaAsP base layer, and the $\mathrm{n}-$ InGaAsP and n-InP joint collector layers, form a doubleheterostructure bipolar transistor. The LD and the HBT share the middle $n$-InP layer which serves as part of the collector of the HBT as well as the bottom confining layer of the laser. The bipolar transistor employs both a wide gap emitter and a wide gap collector [7]. A wide gap emitter ensures high emitter efficiency. A wide gap collector has the advantage of suppressing hole injection from base into the collector under saturation conditions, while allowing the interchangeability of the collector and emitter. To overcome the possible electron repelling effect [8] arising from the abrupt InGaAsP/InP base--collector junction, a thin InGaAsP low bandgap layer was inserted between the InGaAsP base and InP collector layer. Since the structure was grown on an $n^{+}-\operatorname{InP}$ substrate to be compatible with conventional laser technology, the HBT was intended to operate in an emitter-down configuration. 


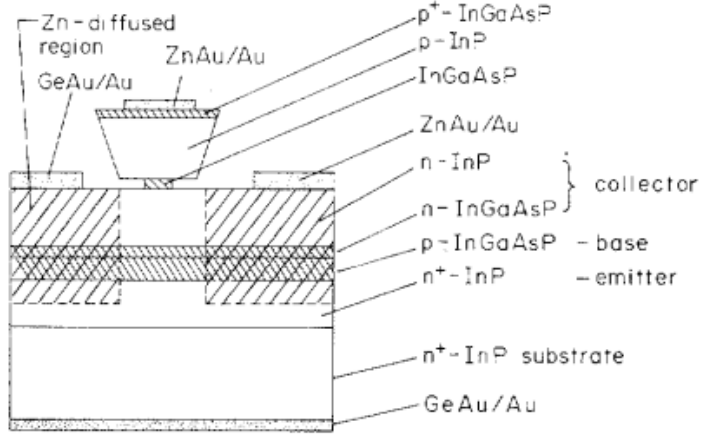

Fig. 1. The schematic structure of a vertically combined DH laser and a heterojunction bipolar transistor

The advantages of fabricating a laser and an HBT in a vertical tandem now become obvious. The technology for making a DH laser and an HBT are compatible in view of the crystal growth as well as the fabrication process. The whole device can be constructed principally by a singlestep crystal growth, which is difficult with a planar configuration [5]. By placing the laser and the HBT vertically close to each other, both optical coupling and electronic interaction [1]-[3], [5] can take place. As a result, new device functions, such as optical or electrooptical switching and bistability (which cannot be expected for constituent discrete optoelectronic devices), can be realized. This is indeed the case for our monolithic device. The utilization of a third dimension (the vertical dimension) also makes the monolithic device more compact.

The monolithic device possesses a $\mathrm{p}-\mathrm{n}-\mathrm{p}-\mathrm{n}$ structure, giving rise to a thyristor-like $I-V$ characteristic as schematically shown in Fig. 2. At low base injection currents, one can see negative differential resistance characteristics which originate from the electronic and/or the optical positive feedback. Depending on the load resistance and the operating conditions (such as the bias voltage across the whole device $V_{c e}$ ), a single device can be operated either as 1) a conventional laser whose output can be controlled either by bias voltage $V_{c e}$ or by base injection current $I_{b}$, 2) an electrooptical bistable device, or 3) an electrooptical switching device. In Fig. 2(a), load lines $(a),(b)$, and $(c)$ show how load resistances select the operation state of the monolithic device. Load lines $\left(a^{\prime}\right),\left(b^{\prime}\right)$, and $\left(c^{\prime}\right)$ [in Fig. 2(b)] show how the job can be done by changing the bias voltage. The device can also be operated as an optical functional device using the HBT as a phototransistor, as pointed out and demonstrated in [1].

\section{Fabrication and Characteristics of the HBT}

In order to study the performance of the InP / InGaAsP / InP double-heterostructure bipolar transistor, a five-layer structure was grown on an $\mathrm{n}^{+}-\mathrm{InP}$ substrate using LPE techniques. Since the characteristics of an HBT greatly depend on the width and the doping concentration in the base region [1], it is important to control them carefully. The species of dopants for base regions also play an important role. In this study, $\mathrm{Zn}$ and $\mathrm{Cd}$ were both tried as p dopants; better performance came from the

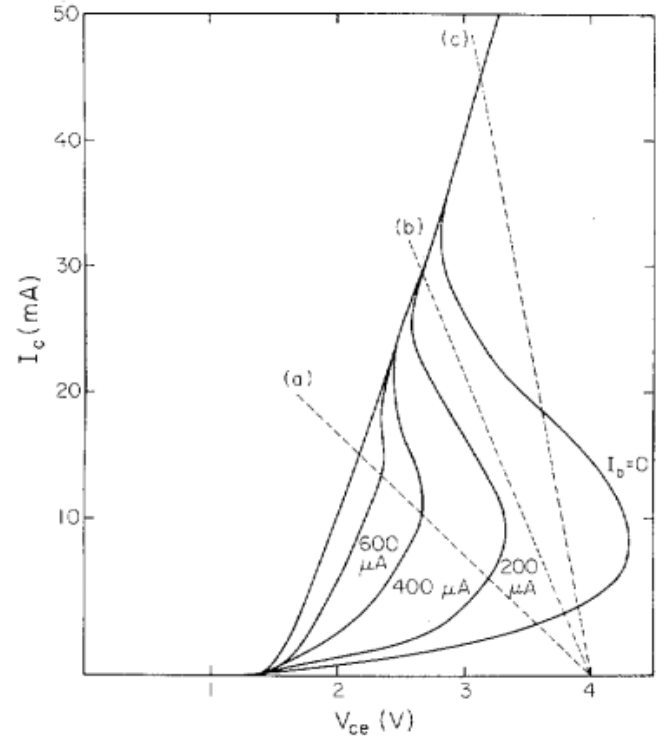

(a)

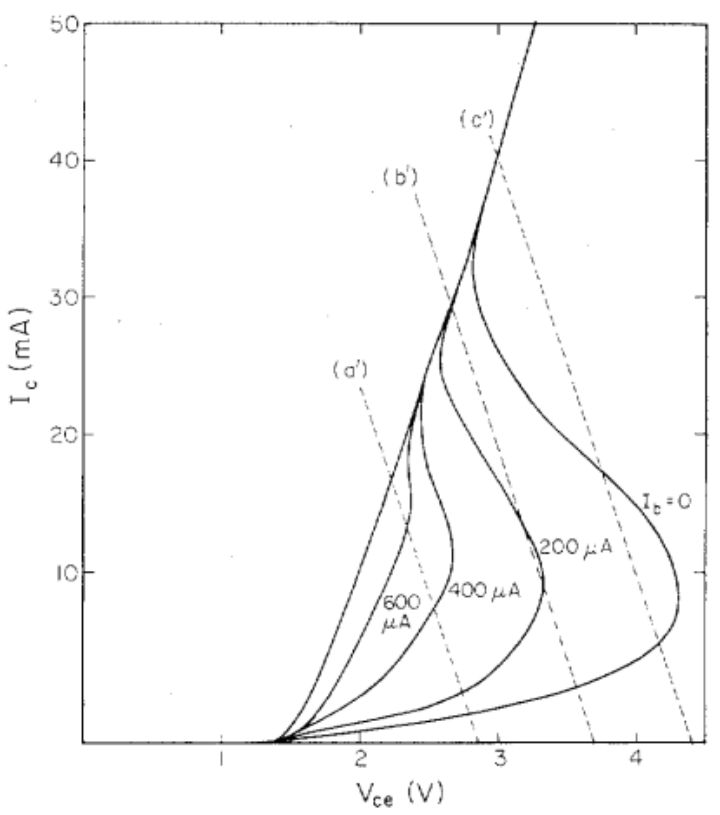

(b)

Fig. 2. (a) $I-V$ characteristics of a monolithic device as a function of base current. Curves $(a),(b)$, and $(c)$ show load-line selection for a basecurrent controlled normal laser, an electrooptical bistable laser, and an electrooptical switching laser. Horizontal: bias voltage across the whole device $V_{c e}$; vertical: collector current $I_{c}$. (b) $I-V$ characteristics of a monolithic device as a function of base current. Curves $\left(a^{\prime}\right),\left(b^{\prime}\right)$, and $\left(c^{t}\right)$ show bias voltage selection for a base current-controlled normal laser, an electrooptical bistable laser, and an electrooptical switching laser. Horizontal: bias voltage across the whole device $V_{c e}$; vertical: collector current $I_{c}$.

Cd-doped devices. The composition, layer thickness, and doping concentration of the five-layer structure are: $\mathrm{n}^{+}-$ InP buffer layer ( $5 \mu \mathrm{m}$ thick, Sn doped to $\sim 10^{18} / \mathrm{cm}^{3}$ ), p-InGaAsP base layer (bandgap wavelength $\lambda_{g}=1.3$ $\mu \mathrm{m}, 0.2-0.4 \mu \mathrm{m}$ thick, $\mathrm{Zn}$ or $\mathrm{Cd}$ doped to $1-3 \times$ $\left.10^{17} / \mathrm{cm}^{3}\right), \mathrm{n}$-InGaAsP layer $\left(\lambda_{g}=1.3 \mu \mathrm{m}, \sim 0.1 \mu \mathrm{m}\right.$ thick, undoped or Sn doped to $\left.\sim 1-3 \times 10^{17} / \mathrm{cm}^{3}\right)$, nInP layer (2-3 $\mu \mathrm{m}$ thick, undoped or Sn doped to 1-3 $\times$ 


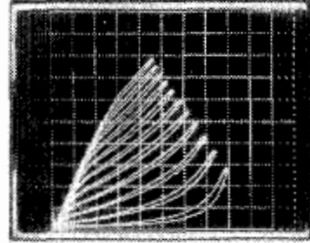

Emitter-down

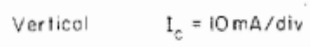

Horizontal $\quad V_{\mathrm{ce}}=500 \mathrm{mV} / \mathrm{div}$

Base Current $I_{t}=20 \mu \mathrm{A} /$ step

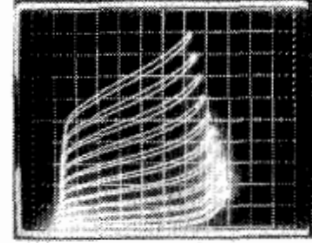

Emitter-up

Base Current $I_{b}=20 \mu \mathrm{A} /$ step

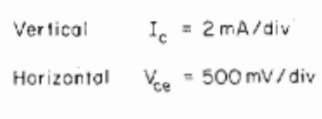

Fig. 3. I- $V$ characteristics of an HBT operated in emitter-down configuration. $V_{c e}$ : voltage across collector and emitter.

$\left.10^{17} / \mathrm{cm}^{3}\right)$, and $\mathrm{n}^{+}-$InGaAsP contact layer $\left(\lambda_{g}=1.1 \mu \mathrm{m}\right.$, $\sim 0.2 \mu \mathrm{m}$ thick, Sn doped to $\sim 2 \times 10^{18} / \mathrm{cm}^{3}$ ). After crystal growth, $\mathrm{Si}_{3} \mathrm{~N}_{4}$ was deposited on the wafer; $10 \mu \mathrm{m}$ mesa stripes and $\sim 15 \mu \mathrm{m}$ openings were defined by standard photolithography followed by selective chemical etching. The etching was stopped in the n-InP collector layer, $\sim 0.5 \mu \mathrm{m}$ away from the $\mathrm{n}$-InGaAsP layer. $\mathrm{A} \mathrm{Zn}$ diffusion was then performed in a sealed ampule at $620^{\circ} \mathrm{C}$ for about $15 \mathrm{~min}$. The $\mathrm{Zn}$ diffusion front penetrated the $\mathrm{p}-\mathrm{InGaAsP}$ base layer and stopped in the $\mathrm{n}^{+}-\mathrm{InP}$ region. A pair of grooves of $5 \mu \mathrm{m}$ width and $\sim 8 \mu \mathrm{m}$ depth were then etched into the $\mathrm{n}^{+}-\mathrm{InP}$ substrate to isolate each individual device. Finally, $\mathrm{GeAu} / \mathrm{Au}, \mathrm{CrAu}$, and $\mathrm{GeAu} / \mathrm{Au}$ were evaporated and annealed to form collector, base, and emitter contact, respectively. To facilitate the measurement, bonding pads were made over polyimide or photoresist which was used to fill up the grooves, giving the device a planar surface (see Fig. 5).

In contrast to the commonly used emitter-up geometry, it was intended that the HBT be operated in an emitterdown configuration. Typical $I-V$ characteristics of the Cddoped HBT were shown in Fig. 3(a). High common emitter current gain and high collector current (which are of key importance in use as a driving circuit for lasers) were achieved. A current gain $\beta$ of about 500 (at $I_{c} \simeq 40$ $\mathrm{mA}, V_{c e} \simeq 3 \mathrm{~V}$ ) and a collector current of over $60 \mathrm{~mA}$ were typical. For some devices, collector currents were driven to as high as $200 \mathrm{~mA}$ (dc). A current gain larger than 1500 (at a collector current of a few $\mathrm{mA}$ ) was also observed. Among devices from the same processed wafer, the performance was quite uniform.

\section{Fabrication of Monolithic Device}

As mentioned in Section II, the monolithic device consisted of seven epi layers. The bottom four layers, i.e., $\mathrm{n}^{+}-\mathrm{InP}$ buffer layer, $\mathrm{p}-\mathrm{InGaAsP}$ base layer, $\mathrm{n}$-InGaAsP, and $n-I n P$ joint collector layers, were described in Section III. The parameters of the top three layers are: undoped InGaAsP active layer $\left(\lambda_{g}=1.3 \mu \mathrm{m}, \sim 0.2 \mu \mathrm{m}\right.$ thick), p-InP upper confining layer (2-3 $\mu \mathrm{m}$ thick, $\mathrm{Zn}$ or Cd doped to $\left.2-5 \times 10^{17} / \mathrm{cm}^{3}\right)$, and $\mathrm{p}^{+}$-InGaAsP cap layer ( $\lambda_{g}=1.2 \mu \mathrm{m}, \sim 0.2 \mu \mathrm{m}$ thick, $\mathrm{Zn}$ or $\mathrm{Cd}$ doped to $\sim 10^{18} / \mathrm{cm}^{3}$ ). The growth temperature for the active layer

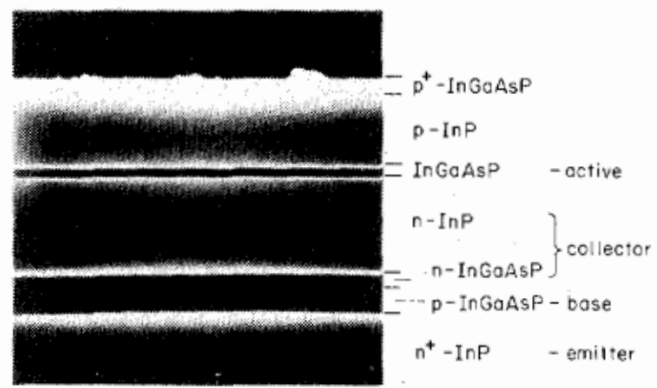

Fig. 4. An SEM view of the seven-LPE-layer structure.

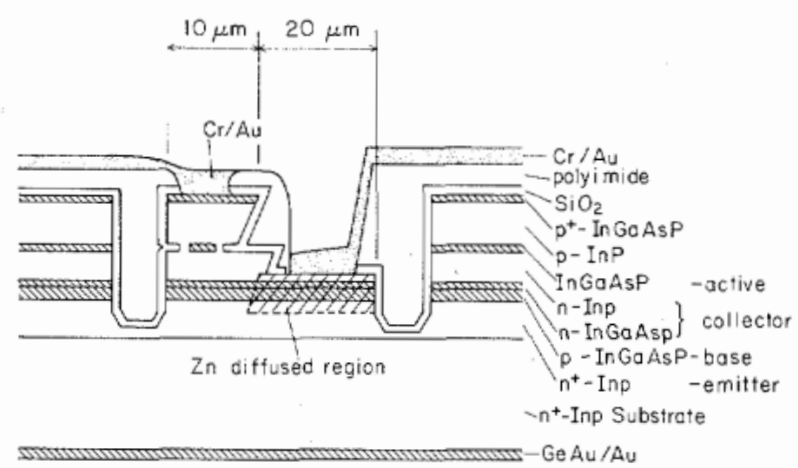

Fig. 5. Cross section of a fabricated monolithic device

was about $630^{\circ} \mathrm{C}$. An SEM view of the grown layers is shown in Fig. 4.

After crystal growth, fabrication of the monolithic device proceeded with laser making. In the first stage of this work, an undercut mesa laser [9] was chosen as a candidate. Not only is the fabrication process of a mesa laser rather simple, it is also easy to obtain very low threshold current. (For a separate study of an undercut mesa laser, $I_{\mathrm{th}} \simeq 9 \mathrm{~mA}$ was obtained.) The most important point is that the fabrication processes of a mesa laser do not involve any serious high-temperature heating which was thought very detrimental to the performance of the monolithic device. The grown wafer was first coated with $\mathrm{SiO}_{2}$ (in a CVD system, at $350-400^{\circ} \mathrm{C}$ ). A $10 \mu \mathrm{m}$ wide laser mesa was then defined in the (011) direction by photolithography and selective wet chemical etching. The etching was stopped in the $\mathrm{n}-\mathrm{InP}$ collector layer, about $0.5 \mu \mathrm{m}$ above the $\mathrm{n}$-InGaAsP layer. The wafer was then $\mathrm{Zn}$ diffused in a sealed ampule $\left(\sim 620^{\circ} \mathrm{C}, \sim 15 \mathrm{~min}\right)$. After diffusion, selective undercutting of the active layer was performed, resulting in a $\sim 2 \mu \mathrm{m}$ wide active region. The individual monolithic devices were then isolated from each other by a pair of deep grooves. Polyimide or photoresist was used to fill up the grooves. Three electrodes for the laser p contact, the base, and emitter of the HBT were made.

In the second stage of this work, a mass transport (MT) laser, which was expected to be more reliable, was chosen to replace the mesa laser. The advantage of involving only a single-step LPE growth remained, although the fabrication of a mass transport laser usually requires high-temperature heating. There was concern that $\mathrm{Zn}$ or $\mathrm{Cd}$ out- 


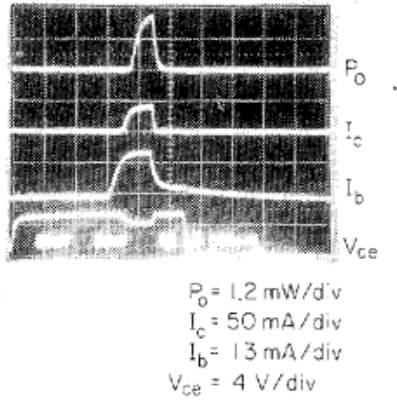

Fig. 6. Oscilloscope traces showing operation of a monolithic mesa laser and $\mathrm{HBT}$ with $\mathrm{Zn}$ as p dopant. From top to bottom: $P_{0}$ : laser power 1.2 $\mathrm{mW}$ per division; $I_{c}$ : collector current, $50 \mathrm{~mA}$ per division; $I_{b}$ : base current, $13 \mathrm{~mA}$ per division; $V_{c e}$ : bias voltage across the monolithic device, $4 \mathrm{~V}$ per division.

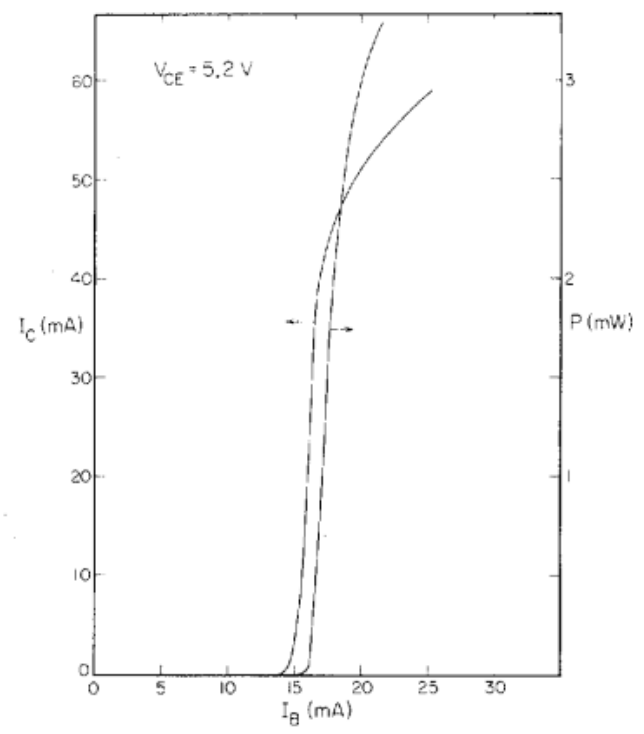

Fig. 7. Collector current and laser output power of a monolithic mesa laser and HBT as a function of base current.

diffusion during the heating period might cause serious junction shift which would degrade device performance; fortunately, the integration of an MT laser with an HBT succeeded with a Cd-doped grown wafer. The fabrication process for this device is similar to that described in the previous paragraph of this section except that a mass transport step was added [9]. The mass transport was carried out at $670-700^{\circ} \mathrm{C}$ for $1-1.5 \mathrm{~h}$ and was performed prior to the $\mathrm{Zn}$ diffusion. In addition, during crystal growth an $\mathrm{n}^{+}-\mathrm{InP}$ anti-diffusion layer (Sn-doped to $\sim 10^{18} / \mathrm{cm}^{3}, 0.5 \mu$ m thick) was inserted between the $\mathrm{n}$ InP collector layer and the laser active layer.

\section{Performance of the Monolithic Device}

Laser operation in a vertical monolithic LD and HBT was realized for the first time with a $\mathrm{Zn}$-doped device. Fig. 6 gives the oscilloscope traces showing one of the device's operation. The device was biased to $V_{c e}=5.2$ V. At base current $I_{b}=16 \mathrm{~mA}$, the collector current $I_{c}$ was $\sim 40 \mathrm{~mA}$ and the laser delivered a light power of $\sim 2$ $\mathrm{mW}$. For this particular device $\beta$ was $\sim 20$ (at $I_{c}=15$ $\mathrm{mA}$ ) and the laser was driven up to $60 \mathrm{~mA}$. At bias volt-

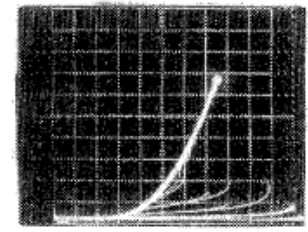

Vertical $I_{C}=5 \mathrm{ma} /$ div

Horizontal $V_{c e}=500$ miv/ oiv

Bose Current $I_{\mathrm{L}}=5 \mu \mathrm{A} / \mathrm{step}$

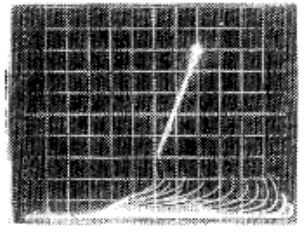

vertical $\quad I_{G}=5 \mathrm{~mA} / \mathrm{div}$

Horizontal $\quad V_{c e}=500 \mathrm{~mW} / \mathrm{div}$

Base Current $I_{t}=2 \mu \mathrm{A} / \mathrm{step}$
Fig. 8. Typical $I-V$ characteristics of a monolithic mesa laser and an HBT with $\mathrm{Cd}$ as $\mathrm{p}$ dopant. Horizontal: bias voltage across the whole device $V_{c e}$; vertical: collector current $I_{c}$.
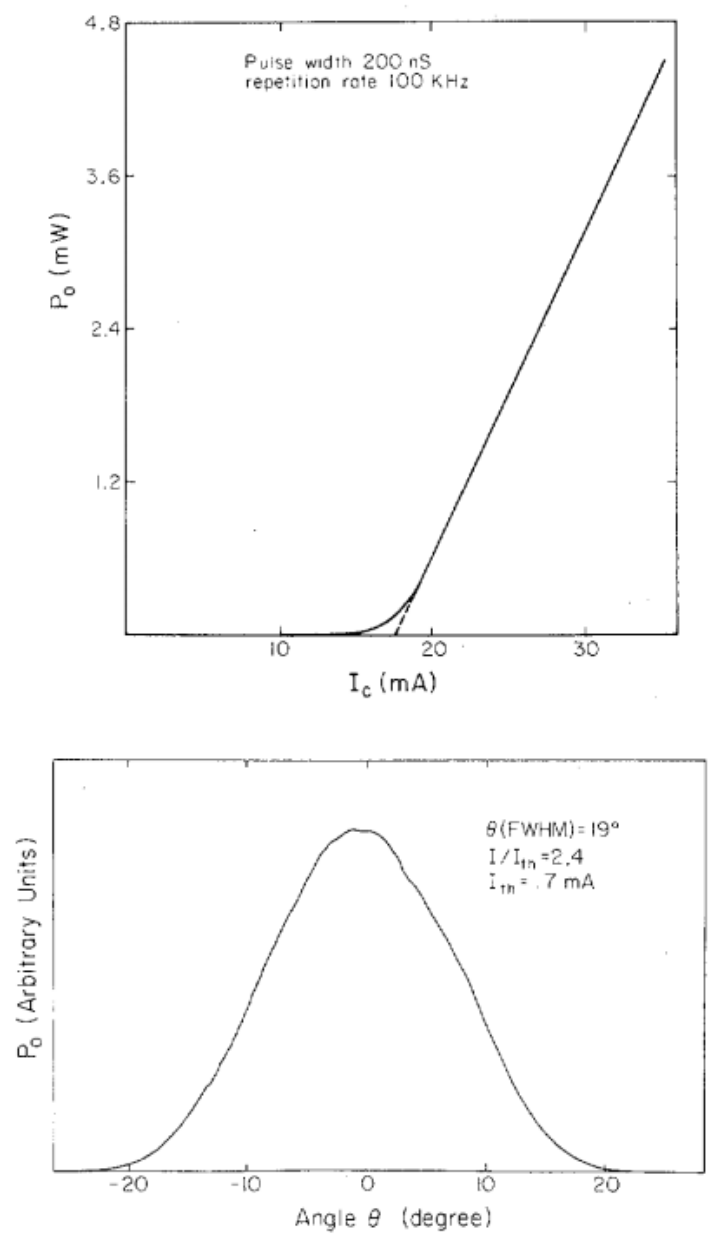

Fig. 9. Light versus collector current characteristic and laser far-field pattern of a monolithic mass transport laser and an HBT.

age $V_{c e}=4 \mathrm{~V}$, the measured laser threshold current was $I_{c}=30 \mathrm{~mA}$. The external quantum efficiency $\left(\Delta P_{0} / \Delta I_{c}\right)$ was $0.23 \mathrm{~mW} / \mathrm{mA}$ per facet. It is interesting to note that the pulsewidths of $I_{c}$ and $P_{0}$ were narrower than that of $I_{b}$. This pulse sharpening can be understood and may be advantageous to the pulse modulation of the laser light. The collector current $I_{c}$ and the output light power $P_{0}$ as a function of base current $I_{b}$ are shown in Fig. 7. Effective control of the laser output via the base current was realized with $\Delta P_{0} / \Delta I_{b}=120$ percent per facet.

Much higher current gain and device uniformity were 


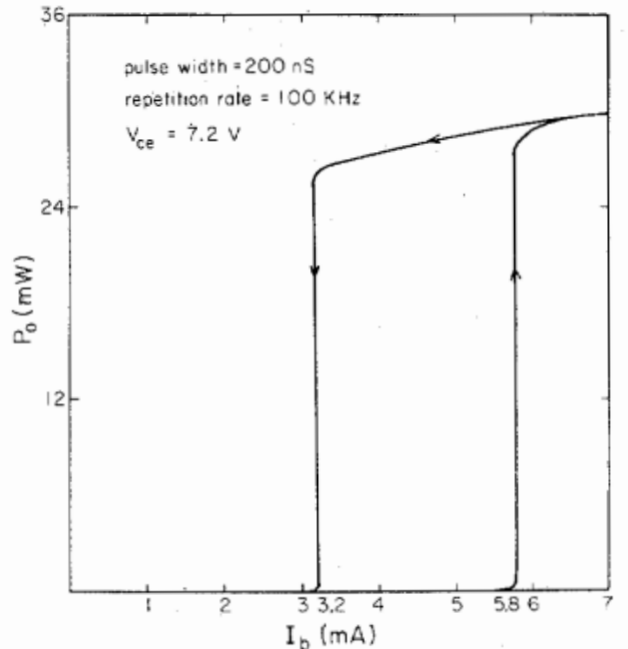

(a)

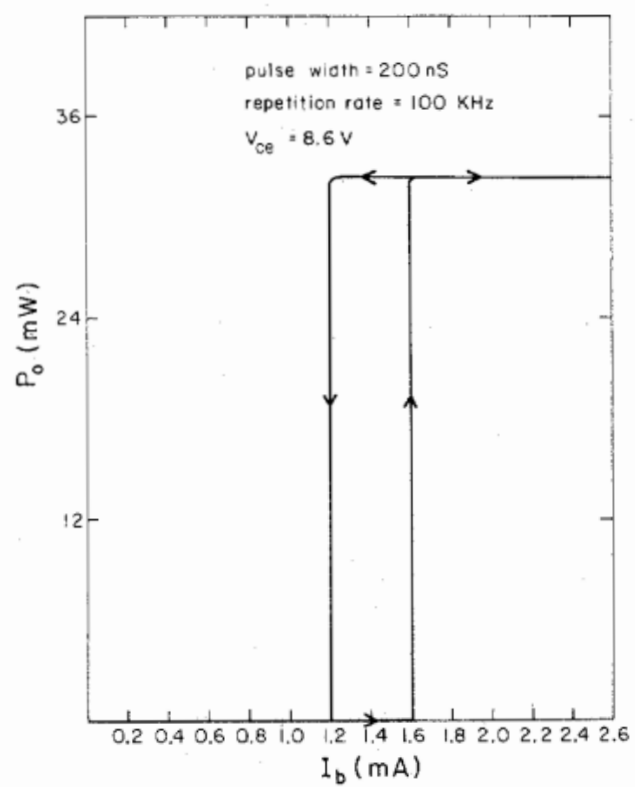

(b)

Fig. 10. (a) Bistable operation of a monolithic device, showing wide dynamic range. (b) Bistable operation of a monolithic device, showing narrow dynamic range.

obtained with Cd-doped devices. Typical $I-V$ characteristics for the Cd-doped monolithic device is shown in Fig. 8.

The monolithic MT laser and HBT (with Cd as a p dopant) showed very promising performance. The threshold current of these lasers were in the range of $20-40 \mathrm{~mA}$ (for cavity length of $\sim 250 \mu \mathrm{m}$ ), with the lowest one being $17 \mathrm{~mA}$. The laser operated in a single transverse mode. Laser output power as a function of collector current and a measured far-field pattern of the laser are shown in Fig. 9. $\mathrm{CW}$ operation was also achieved, despite the $\mathrm{p}$ side up configuration. The external quantum efficiency of the MT laser was typically $0.25 \mathrm{~mW} / \mathrm{mA}$ per facet. Under pulse operation conditions, the collector current can be driven to over $300 \mathrm{~mA}$. An output light power of over $30 \mathrm{~mW}$ was observed.
Electrooptical bistable laser operation and electrooptical switching were successfully demonstrated. These operation states take advantage of the electronic as well as optical feedback (optical coupling is caused by the leakage of part of the optical radiation from the laser into the base region of HBT). As a matter of fact, most of the monolithic devices exhibited more or less switching behavior under high bias voltage. Fig. 10 presents light versus base current characteristics of two of the bistable devices. The dynamic range of the bistability depends on the details of the device $I-V$ characteristic and is also a function of bias voltage. For the devices shown in Fig. 10 , a base injection current of a few $\mathrm{mA}[1.6 \mathrm{~mA}$ in the case of Fig. 10(b)] switched the device to an "on" state with a collector current of $\sim 200 \mathrm{~mA}$ (not shown in the figure) and a laser light power of over $30 \mathrm{~mW}$.

\section{CONClusions}

High-performance $1.3 \mu \mathrm{m}$ InGaAsP/InP double-heterostructure mesa lasers and mass transport lasers have been monolithically combined with high-performance InGaAsP/InP double-heterostructure bipolar transistors in a vertical configuration. Laser thresholds current as low as $17 \mathrm{~mA}$, coherent output light power in excess of 30 $\mathrm{mW}$, current gain of over 500 , and collector current as high as $200 \mathrm{~mA} \mathrm{(dc)} \mathrm{were} \mathrm{achieved.} \mathrm{Three} \mathrm{operation}$ modes, base current-controlled normal laser operation, electrooptical bistable operation, and electrooptical switching, have been realized.

Potential high-speed operation was taken into account in the design and fabrication of the monolithic devices by using appropriate laser types and small device dimensions. High-speed modulation and high-speed switching are, therefore, expected. Using these monolithic devices, a number of optical functions with coherent light output such as optical amplification, switching, and wavelength conversion are made possible. A combination of electronic and optical control of the monolithic device's operation states may result in interesting applications. For example, the device can be either optically biased [2] and electronically modulated from the base terminal or electrically biased (at fixed $V_{c e}$ and fixed $I_{b}$ ) and optically modulated-or even both.

\section{ACKNOWLEDGMENT}

The author gratefully acknowledge Dr. B. Chang, D. Armstrong, W. Marshall, J. Mercado, and R. Wong for their assistance.

\section{REFERENCES}

[1] A. Sasaki, K. Matsuda, Y. Kimura, and S. Fugita, "High-current InGaAsP-InP phototransistors and some monolithic optical devices," IEEE Trans. Electron Devices, vol. ED-29, p. 1382, Sept. 1982.

[2] A. Sasaki, M. Taneya, H. Yano, and S. Fujita, "Optoelectronic integration device with light amplification and optical bistability," IEEE Trans. Electron Devices, vol. ED-31, p. 805, June 1984.

[3] A. Sasaki, H. Yano, S. Fujita, and Y. Takeda, "Integrated optical devices of InGaAsP / InP heterojunction phototransistor and inner stripe light-emitting diode," J. Lightwave Technol., vol. LT-3, p. 1264, Dec. 1985 
[4] J. Shibata, I. Nakao, Y. Sasai, S. Kimura, N. Hase, and H. Serizawa, "Monolithic integration of an InGaAsP/InP laser diode with heterojunction bipolar transistor," Appl. Phys. Lett., vol. 45, p. 19l, Aug. 1984.

[5] H. Grothe and W. Proebster, "Monolithic integration of InGaAsP / InP LED and transistor-A light coupled bistable electro-optical device," Electron. Lett., vol. 19, p. 194, Mar. 1983.

[6] S. Margalit and A. Yariv, Semiconductor and Semimetal, vol. 22, part E, ch. 2, "Integrated electronic and photonic devices," Orlando, FL: Academic, 1985.

[7] H. Kroemer, "Heterostructure bipolar transistors and integrated circuits," Proc. IEEE, vol. 70, p. 13, 1982.

[8] L. M. Su, N. Arote, R. Kaumanns, and H. Schroeter, "NPnN double heterojunction bipolar transistor on InGaAsP/InP," Appl. Phys. Lett., vol. 47, p. 1115, July 1985.

[9] U. Koren, T. R. Chen, C. Harder, A. Hasson, K. L. Yu, L. C. Chiu, S. Margalit, and A. Yariv, "InGaAsP / InP undercut mesa laser with planar polymide passivation," Appl. Phys. Lett., vol. 42, p. 403, 1983.

[10] T. R. Chen, L. C. Chiu, K. L. Yu, U. Koren, A. Hasson, S. Margalit, and A. Yariv, "Low threshold InGaAsP terrace mass transport laser on semi insulating substrate," Appl. Phys. Lett., vol. 41, p. 1115,1982 .

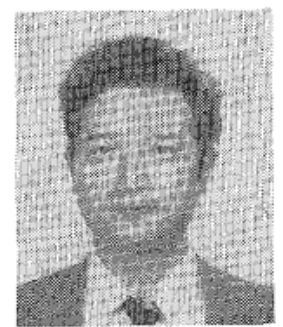

T. R. Chen was born in Changshar, China. He graduated from the Department of Physics, Wuhan University, Wuhan, China, in 1962.

Shortly after graduation he joined the faculty of Chengdu Institute of Radio Engineering, Chengdu, China, becoming a Full Professor in 1984. He was a Visiting Associate at the California Institute of Technology, Pasadena, for the period 1980-1983 and again from 1985 until the present time. He was a Visiting Professor with the Department of Electrical Engineering, University of Illinois, Urbana-Champaign, during the summer of 1981. His research interests have been in the field of quantum electronics, charge density waves, guided-wave optics, semiconductor lasers, and optoelectronic integration. $\mathrm{He}$ is the author or coauthor of three books (in Chinese) and 40 academic journal papers.
Katsuyuki Utaka (S'80-M'81), for a photograph and biography, see this issue, p. 821 .

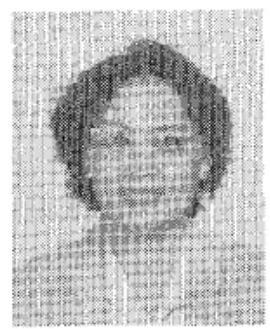

Yuhua Zhuang was born in Chengdu, China. She graduated from the Department of Applied Mcchanics, Chengdu University of Science and Technology, Chengdu, in 1962.

After graduation she joined the faculty of the Physics Department, Chengdu Institute of Radio Engineering. She is presently doing research in semiconductor optoclectronics with the Department of Applied Physics, California Institute of Technology, Pasadena.

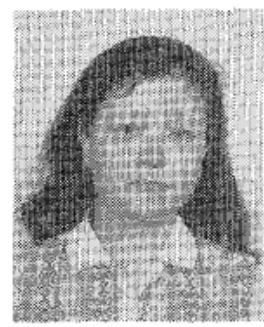

Ya-Yun Liu received the B.S. degree in semiconductor materials and devices from Tsinghua University, Beijing, China, in 1968.

She was employed as an engineer for an integrated circuit manufacturing company from 1968 to 1978. She joined the Chinese Academy of Railroad Sciences in 1979 and was engaged in research of semiconductor-controlled rectifiers until 1984. From February 1985 to the present she has been working on semiconductor lasers with the tute of Technology, Pasadena Department of Applied Physics, California Insti-

Amnon Yariv (S'56-M'59-F'70), for a photograph and biography, see p. 399 of the April 1987 issue of this Journal. 